\title{
THE PHASE SPACE FOR CONFORMAL PARTICLE WITH ZERO MASS AND SPIRALITY AND ITS RELATION WITH THE KEPLER'S PROBLEM
}

\author{
ALEXANDER B. YANOVSKI
}

Communicated by Charles-Michel Marle

Abstract. We show that the two orbits in the coadjoint representation of the group $\mathrm{SO}(4,2)$, the one, related with the Kepler's problem, and the other - modelling the phase space of a conformal particle with zero mass and spirality zero, coincide.

\section{Introduction}

The the idea for the present work arose naturally in the development of two important trends in the Classical Mechanics and Differential Geometry. The first one is related with the so-called Kirillov-Kostant-Souriau theorem, that allows to identify connected symplectic spaces having sufficiently large dynamical group with orbits in the coadjoint representation of these groups, $[12,21]$. The second is the circle of ideas related to the geometric quantization $[13,21]$. If the symplectic manifold is given, we expect to quantize some subalgebra of functions (usually finite dimensional) and naturally such a subalgebra is associated with some Lie group. Then the idea of the Kirillov-Kostant-Souriau theorem can be developed in somewhat opposite direction, that is, to start with certain Lie algebra $\mathcal{G}$, to which corresponds a connected Lie group $G$, and then using it, to find a phase space having $G$ as dynamical group. Natural candidate for it will be some orbit in the coadjoint representation of $G$. One usually imposes also the condition that such $G$ must contain the Poincaré or the Galilean group in order to be able to give physical interpretations. It is clear that the above procedure can be performed even for quantum systems that have no classical counterparts in the usual sense. The phase space (symplectic manifold) will be simply an orbit in the coadjoint representation of the group, picked up by fixing the values of some important physical observables (such as mass for example), [21]. It is natural to expect, that some of the spaces, obtained in this way may coincide with some classical phase spaces. The Hydrogen atom problem (Kepler's problem) turned 
out to be an example of that situation. On the first place, the group $\mathrm{SO}(4,2)$ and its coadjoint orbit has been applied already in the geometric quantization of the Hydrogen atom problem for negative energies, see [13, 19]. Then in [15, 23] it was remarked that on the quantum level, the representation describing conformal particle with $m=0, \lambda=0$ (mass zero and spirality zero), is the well known ladder representation of the group $\mathrm{SU}(2,2)$ (the dynamical group of the quantum problem). As $\mathrm{SU}(2,2)$ and $\mathrm{SO}(4,2)$ locally coincide $\mathrm{SU}(2,2)$ is the universal cover of $\mathrm{SO}(4,2))$ one can ask if both problems coincide at the classical level, that is, if they have identical phase spaces. In the present work we shall prove that this is indeed the case. As the classical problems for the Hydrogen atom and the Kepler's problem are identical, we use both these names as synonyms. Also, in both cases it is enough to consider the connected component of the unity, that is $\mathrm{SO}_{e}(4,2)$, since we want the resulting phase spaces to be connected.

Some words about the terminology and notation. Let $G$ be a Lie group acting canonically and transitively on the left on the symplectic manifold $(\mathcal{M}, \omega)$, where $\omega$ is the symplectic form. We call usually $(\mathcal{M}, \omega)$ phase space and we denote the action of $G$ by $G \ni g \mapsto l_{g}$, where $l_{g}$ is a symplectic diffeomorphism of $\mathcal{M}$, that is $l_{g}^{*} \omega=\omega$. Such a group is called a dynamical group. If we have dynamics defined by a Hamiltonian function $H$ and the dynamical group preserves the Hamiltonian $l_{g}^{*} H=H$, for $g \in G$, we call it a symmetry group.

As well known, see for example $[1,20]$, if $G$ is a dynamical group then the momentum map (or simply momentum) is a function $\mu: \mathcal{M} \rightarrow \mathcal{G}^{*}$ having the property

$$
\mathrm{d}\langle\mu(x), \xi\rangle=i_{\widetilde{\xi}} \omega
$$

for arbitrary $\xi \in \mathcal{G}$, where $\mathcal{G}$ is the Lie algebra, corresponding to $G, \widetilde{\xi}$ is the fundamental field defined by the element $\xi \in \mathcal{G}$ and the action of $G$ and $\langle$,$\rangle denotes the$ canonical pairing between the algebra $\mathcal{G}$ and the co-algebra $\mathcal{G}^{*}$. The existence of momentum is equivalent to the existence of $m$ scalar functions $\mu_{i}, i=1,2, \ldots, m$ such that

$$
\mathrm{d}\left\langle\mu_{i}(x), \xi\right\rangle=i_{\widetilde{\xi}_{i}} \omega
$$

where $\left\{\xi_{i}\right\}_{i=1}^{m}$ is some fixed basis of $\mathcal{G}$. Of course, when $G$ is a symmetry group, all $\mu_{i}$ are integrals of motion. If $\mathcal{M}$ is connected and if certain cohomology class for the algebra $\mathcal{G}$, constructed through the action of of $G$, is trivial, $x \mapsto \mu(x)$ is an equivariant map between the space $(\mathcal{M}, \omega)$ and an orbit in the coadjoint representation of $G$. In this case

$$
\left\{\mu_{i}, \mu_{j}\right\}=c_{i j}^{k} \mu_{k}
$$


where $\left[\xi_{i}, \xi_{j}\right]=c_{i j}^{k} \xi_{k}$. We shall always assume that this is the case, because all our Lie algebras will be semisimple. For such algebras, according to the classical Whitehead's lemma the first and second cohomology spaces are zero in arbitrary representation. Since the cohomology class, we were speaking about in above, belongs to the second cohomology space with respect to the trivial representation, see [20], it will be trivial. Besides, the question about this class will not appear at all, as we shall use (3) reversely, that is we shall find explicitly such functions $\mu_{i}$ having the same Poisson brackets as the generators of the algebra $\mathcal{G}$ of some connected Lie group $G$, specifying the orbit and hence the action of $G$.

The fact that $\mathrm{SO}_{e}(4,2)$ is a dynamical group for the Kepler's problem (i.e., the Hydrogen atom problem) and symplectic imbedding into orbits of the coadjoint representation of $\mathrm{SO}_{e}(4,2)$ (or its universal cover $\mathrm{SU}(2,2)$ ) is in fact well known and the properties of generators of $\mathfrak{s o}(4,2) \sim \mathfrak{s u}(2,2)$ relative to the Kepler's problem has been extensively studied, both on classical and quantum level, [3, $6,7,10,16,24]$. The group aspects of the Kepler's problem can be found in $[9,21]$ while a review and exposition of both classical results and the most recent ones can be found in [4]. In [14] the group $\mathrm{SU}(2,2)$ has been used to study the perturbations of the Kepler's dynamics, the same group is applied also to the study of a related dynamics (the so-called MIC-Kepler Problem) in [17] and [26], see also [11] and the references therein.

The $\mathrm{SO}_{e}(4,2)$ orbit, as a manifold defined by some relations, usually is found indirectly. There can be some variations, but usually the first step is to consider the famous symplectic immersion $F$ into the space $\left(T^{+} \mathbb{S}^{3}, \omega_{S}\right)$, which can be found using the $\mathrm{O}(4)$ momentum function. Here $T^{+} \mathbb{S}^{3}$ is the tangent bundle of the 3dimensional sphere $\mathbb{S}^{3}$, with the zero section removed and the symplectic structure $\omega_{S}$ is obtained restricting the symplectic structure of $\mathbb{R}^{6}$ where $T^{+} \mathbb{S}^{3}$ is canonically embedded. The map $F$ was found and applied to regularize the Hamiltonian flow of the Kepler's problem [18], and there are some different variants of that result, see $[4,5]$. Then one uses the symplectic immersion of $\left(T^{+} \mathbb{S}^{3}, \omega_{S}\right)$ into an orbit of the coadjoint representation of $\mathrm{SO}_{e}(4,2)$ (or its universal cover $\mathrm{SU}(2,2)$ ). Finally, as a result, the phase space for the Kepler's problem is symplectically immersed into the corresponding orbit. We show how all this can be done directly. In this we follow some of the calculations of [2], where it has been found an algebra of functions on $\mathcal{M}$, identical to the algebra $\mathfrak{s o}(4,1)$ (with respect to the Poisson bracket). We follow closely the method of [2] and do the same for $\mathfrak{s o}(4,2)$ (the result is known, but we want to sketch the method, besides there are some instructive points in the calculation). This we do in Section 2. Next we find the orbit directly and hope that the way we obtained it is new. Finally, in Section 4 
we calculate another orbit, corresponding to a conformal particle of zero mass and spirality zero and show that it is identical to that, obtained for the Kepler's problem. We believe that this result is also original.

\section{The Group $S O_{e}(4,2)$ as a Dynamical Group for the Kepler's Problem. The Momentum Map}

In what follows we fix the constants for the Hydrogen atom (Kepler's problem) be equal to one. Thus, the Hamiltonian function will be

$$
H=\frac{1}{2} p^{2}-\frac{1}{r}, \quad \boldsymbol{r}, \boldsymbol{p} \in \mathbb{R}^{3}, \quad r=\|\boldsymbol{x}\|, \quad p=\|\boldsymbol{p}\| .
$$

Also, we consider the Kepler's problem for negative energies, that is by definition the phase space $\left(\mathcal{M}, \omega_{3}\right)$ is

$$
\mathcal{M}=\left\{(\boldsymbol{x}, \boldsymbol{p}) \in \mathbb{R}^{6} ; H(\boldsymbol{x}, \boldsymbol{p})<0\right\}, \quad \omega_{3}=\mathrm{d} p_{i} \wedge \mathrm{d} q^{i} .
$$

(In the above formulae summation over repeated indices is implied). One canonical action of a Lie group on $\mathcal{M}$ is well known - the action of the group of rotations $\mathrm{SO}_{e}(3): l_{g}(\boldsymbol{x}, \boldsymbol{p})=(g \boldsymbol{x}, g \boldsymbol{p}), \quad g \in \mathrm{SO}_{e}(3)$. If we choose in $\mathfrak{s o}(4)$ the basis $\left(I_{i}\right)_{k l}=\epsilon_{i j k}$, where $\epsilon_{i j k}$ is the Levi-Cevita symbol, then the momentum functions $\mu_{i}$ are the components of the angular momentum $L_{i}=(\boldsymbol{x} \times \boldsymbol{p})_{i}$. Of course, $\mathrm{SO}_{e}(3)$ is a symmetry group for the Kepler's problem.

The above symmetry group can be extended to $\mathrm{SO}_{e}(4)$, as suggested by the existence of another vector first integral - the so-called Runge-Lenz-Laplace vector

$$
\boldsymbol{A}=(-2 H)^{-\frac{1}{2}}\left[\left(\frac{1}{r}-p^{2}\right) \boldsymbol{x}+u \boldsymbol{p}\right], u=\langle\boldsymbol{x}, \boldsymbol{p}\rangle=x_{i} p_{i}
$$

The momentum map $\mu_{i j}=-\mu_{j i}$ of $\mathrm{SO}_{e}(4)$ is also well known

$$
\left(\mu_{14}, \mu_{24}, \mu_{34}\right)=\left(A_{1}, A_{2}, A_{3}\right), \quad\left(\mu_{32}, \mu_{13}, \mu_{21}\right)=\left(L_{1}, L_{2}, L_{3}\right)
$$

where the functions $\mu_{i j}, 1 \leq i, j \leq 4$ correspond to the standard basis $I_{i j}=$ $e_{i j}-e_{j i}$, (here and below $\left(e_{i j}\right)_{m}^{n}=\delta_{i}^{m} \delta_{j}^{n}$ ). As $\mathfrak{s o}(4) \sim \mathfrak{s o}(3) \oplus \mathfrak{s o}(3)$, the first summand gives rise to the angular momentum $\boldsymbol{L}$ and the second to $\boldsymbol{A}$.

We shall take the functions we have already and shall try to add to them other ones in order to obtain the algebra of the group $\mathrm{SO}_{e}(4,2)$, (the connected component of the linear group preserving the symmetric bilinear form with signature ++++ -- ). Thus we find a larger (dynamical) group, that will be no more a symmetry group. 
Let us cast the commutation relations of $\mathfrak{s o}(4,2)$ in the form

$$
\begin{array}{lllll}
{[\boldsymbol{L}, \boldsymbol{L}]=\boldsymbol{L},} & {[\boldsymbol{A}, \boldsymbol{A}]=\boldsymbol{L},} & {[\boldsymbol{B}, \boldsymbol{B}]=-\boldsymbol{L},} & {[\boldsymbol{L}, \boldsymbol{A}]=\boldsymbol{A},} & {[\boldsymbol{L}, \boldsymbol{B}]=\boldsymbol{B}} \\
{[\boldsymbol{L}, \boldsymbol{C}]=\boldsymbol{C},} & {[\boldsymbol{C}, \boldsymbol{C}]=-\boldsymbol{L},} & {[\boldsymbol{A}, \boldsymbol{C}]=R,} & {[\boldsymbol{A}, \boldsymbol{B}]=Q,} & {[\boldsymbol{C}, \boldsymbol{B}]=S} \\
{[\boldsymbol{L}, Q]=0,} & {[\boldsymbol{L}, R]=0,} & {[\boldsymbol{L}, S]=0} & {[R, \boldsymbol{A}]=\boldsymbol{C},} & {[S, \boldsymbol{A}]=0} \\
{[Q, \boldsymbol{A}]=\boldsymbol{B},} & {[Q, \boldsymbol{B}]=\boldsymbol{A},} & {[Q, \boldsymbol{B}]=0,} & {[S, \boldsymbol{B}]=\boldsymbol{C},} & {[Q, \boldsymbol{C}]=0} \\
{[R, \boldsymbol{C}]=\boldsymbol{A},} & {[S, \boldsymbol{C}]=-\boldsymbol{B},} & {[R, Q]=S,} & {[R, S]=Q,} & {[S, Q]=R}
\end{array}
$$

where

$$
\begin{gathered}
\boldsymbol{L}=\left(I_{32}, I_{13}, I_{21}\right), \quad Q=I_{54} \\
\boldsymbol{A}=\left(I_{14}, I_{24}, I_{34}\right), \quad R=I_{64} \\
\boldsymbol{B}=\left(I_{15}, I_{25}, I_{35}\right), \quad S=I_{56} \\
\boldsymbol{C}=\left(I_{16}, I_{26}, I_{36}\right), \\
I_{i j}=\gamma_{i} e_{i j}-\gamma_{j} e_{j i}, \quad \gamma_{i}= \begin{cases}1 & i=1,2,3,4 . \\
-1 & i=5,6 .\end{cases}
\end{gathered}
$$

In the above we have used the notations

$$
\begin{array}{lll}
{[\boldsymbol{A}, \boldsymbol{B}]=\boldsymbol{C}} & \text { means } & {\left[A_{i}, B_{j}\right]=\epsilon_{i j k} C_{k}} \\
{[\boldsymbol{A}, \boldsymbol{B}]=Q} & \text { means } & {\left[A_{i}, B_{j}\right]=\delta_{i j} Q} \\
{[S, \boldsymbol{B}]=\boldsymbol{C}} & \text { means } & {\left[S, B_{i}\right]=C_{i} .}
\end{array}
$$

(No summation over repeated indices for the formulae for the generators).

The above form is chosen for the following reason. It is known that the condition $\{\boldsymbol{L}, f\}=0$ (here and below we use the same letters for the generators of the algebra and the corresponding momentum functions) is necessary and sufficient in order that the function $f$ be a scalar function (transforms as scalar with respect to the rotational group) and $\{\boldsymbol{L}, \boldsymbol{A}\}=\boldsymbol{A}$ is is necessary and sufficient in order that the function $\boldsymbol{A} \equiv\left(A_{1}, A_{2}, A_{3}\right)$ be a vector function (transforms as vector). Thus those relations which contain $L$ will be satisfied automatically if $\boldsymbol{A}, \boldsymbol{B}, \boldsymbol{C}$ are vector functions and $Q, R, S$ are scalar functions. $\mathrm{A} \mathrm{SO}_{e}(3)$-scalar function depends on $\boldsymbol{x}$ and $\boldsymbol{p}$ only through $r=\|\boldsymbol{x}\|,\|\boldsymbol{p}\|$ and $u=\langle\boldsymbol{x}, \boldsymbol{p}\rangle$. We find it more convenient to assume that this dependence is through $r, u$ and $H=\frac{1}{2} p^{2}-\frac{1}{r}$. From the other side, every vector function can be written into the form

$$
\boldsymbol{B}=f \boldsymbol{x}+g \boldsymbol{p}+h(\boldsymbol{x} \times \boldsymbol{p})
$$

where $f, g, h$ are some scalar functions. But, if we assume the above general form it can be checked that in general $\{\boldsymbol{B}, \boldsymbol{B}\} \neq w \boldsymbol{L}$, (with some scalar function $w$ ). 
On the contrary, when $h=0$ the bracket is proportional to $\boldsymbol{L}$. For that reason we assume that $\boldsymbol{A}, \boldsymbol{B}, \boldsymbol{C}$ have the form

$$
\boldsymbol{A}=f \boldsymbol{x}+g \boldsymbol{p}, \quad \boldsymbol{B}=F \boldsymbol{x}+G \boldsymbol{p}, \quad \boldsymbol{C}=F^{\prime} \boldsymbol{x}+G^{\prime} \boldsymbol{p} .
$$

For example, if $\boldsymbol{A}$ is the Runge-Lenz-Laplace vector, we have

$$
f=N\left(r^{-1}-p^{2}\right), \quad g=u N, \quad N=(-2 H)^{-\frac{1}{2}} .
$$

We can pass now to our task. If we look into the commutation relations of $\mathfrak{s o}(4,2)$ it is not difficult to see that the algebras, spanned by the sets $(\boldsymbol{L}, \boldsymbol{A}, \boldsymbol{B}, Q)$ and $(\boldsymbol{L}, \boldsymbol{A}, \boldsymbol{C}, R)$ are isomorphic, that is (if we write instead of commutators the Poisson brackets) we obtain two identical sets of equations
1) $\{\boldsymbol{L}, \boldsymbol{L}\}=\boldsymbol{L}$
$\left.1^{\prime}\right) \quad\{\boldsymbol{L}, \boldsymbol{L}\}=\boldsymbol{L}$
2) $\{\boldsymbol{L}, \boldsymbol{A}\}=\boldsymbol{A}$
$\left.2^{\prime}\right) \quad\{\boldsymbol{L}, \boldsymbol{A}\}=\boldsymbol{A}$
3) $\{\boldsymbol{L}, \boldsymbol{B}\}=\boldsymbol{B}$
$\left.3^{\prime}\right) \quad\{\boldsymbol{L}, \boldsymbol{B}\}=\boldsymbol{B}$
4) $\{\boldsymbol{A}, \boldsymbol{A}\}=\boldsymbol{L}$
$\left.4^{\prime}\right) \quad\{\boldsymbol{A}, \boldsymbol{A}\}=\boldsymbol{L}$
5) $\{\boldsymbol{A}, \boldsymbol{B}\}=Q$
$\left.5^{\prime}\right) \quad\{\boldsymbol{A}, \boldsymbol{C}\}=R$
6) $\{\boldsymbol{A}, Q\}=-\boldsymbol{B}$
$\left.6^{\prime}\right) \quad\{\boldsymbol{A}, R\}=-\boldsymbol{C}$
7) $\{\boldsymbol{B}, \boldsymbol{B}\}=-\boldsymbol{L}$
$\left.7^{\prime}\right)$
$\{\boldsymbol{C}, \boldsymbol{C}\}=-\boldsymbol{L}$
8) $\{\boldsymbol{B}, Q\}=-\boldsymbol{A}$
$\left.8^{\prime}\right) \quad\{\boldsymbol{C}, R\}=-\boldsymbol{A}$
9) $\{\boldsymbol{L}, Q\}=0$
$\left.9^{\prime}\right) \quad\{\boldsymbol{L}, R\}=0$.

It follows that if we find a solution for the system 1) - 9) it can be used also for the second system $\left.\left.-1^{\prime}\right)-9^{\prime}\right)$. As we have mentioned, if $\boldsymbol{A}, \boldsymbol{B}$ are vector functions and $Q$ scalar function, then 1), 2), 3), 9) are satisfied. Therefore, we need to satisfy only the rest of the equations. It turns out however that only a part of them are independent.

Proposition 1. The equations 6) and 8) from the system (12) are satisfied identically if the rest of the equations are satisfied.

(The proof is quite straightforward, but long and cumbersome and we omit it).

Let us consider now the equations 4), 5) and 7) in (12). We want to obtain an algebra larger than $\mathfrak{s o}(4)$ and for that reason we assume that $\boldsymbol{L}$ is the angular momentum and $\boldsymbol{A}$ is the Runge-Lenz-Laplace vector. Then we must find $F, G$ and $F^{\prime}, G^{\prime}$. It can be shown that 4), 5) and 7) force the following form of $F, G$

$$
\begin{aligned}
& F=r^{-1} a_{1} \sin \frac{u}{N}+r^{-1} b_{1} \cos \frac{u}{N} \\
& G=-\left(N^{-1} r b_{1}+a_{1}\right) \sin \frac{u}{N}+\left(N^{-1} r a_{1}+u b_{1}\right) \cos \frac{u}{N}
\end{aligned}
$$


where $a_{1}, b_{1}$ depend on $(\boldsymbol{x}, \boldsymbol{p})$ only through $H$. Quite analogously, we get $\boldsymbol{C}$ $=F^{\prime} \boldsymbol{x}+G^{\prime} \boldsymbol{p}$, where

$$
\begin{aligned}
& F^{\prime}=r^{-1} a_{2} \sin \frac{u}{N}+r^{-1} b_{2} \cos \frac{u}{N} \\
& G^{\prime}=-\left(N^{-1} r b_{2}+a_{2}\right) \sin \frac{u}{N}+\left(N^{-1} r a_{2}+u b_{2}\right) \cos \frac{u}{N} .
\end{aligned}
$$

Let us substitute what we have obtained in the equations $\{\boldsymbol{B}, \boldsymbol{B}\}=-\boldsymbol{L}$. After some calculations we get that they are equivalent to a single equation: $a_{1}^{2}+b_{1}^{2}=$ $c_{1}+N^{2}$, where $c_{1}$ is some constant. Naturally, we have also $a_{2}^{2}+b_{2}^{2}=c_{2}+N^{2}$ with some constant $c_{2}$. The equations that rest to be satisfied are

$$
\begin{array}{ll}
\text { 1) } & \{\boldsymbol{C}, \boldsymbol{B}\}=S \\
\text { 2) } & \{S, \boldsymbol{B}\}=\boldsymbol{C} \\
\text { 3) } & \{\boldsymbol{C}, S\}=\boldsymbol{B} \\
\text { 4) } & \{\boldsymbol{C}, Q\}=0 \\
\text { 5) } & \{\boldsymbol{B}, R\}=0 \\
\text { 6) } & \{R, Q\}=S \\
\text { 7) } & \{S, Q\}=R \\
\text { 8) } & \{\boldsymbol{A}, S\}=0 .
\end{array}
$$

The proof of the following proposition, though cumbersome, is proved by direct calculations and we give only the result.

Proposition 2. In the system (15) only the equations 1), 2), 3) are independent, that is, if they are satisfied, the rest are satisfied identically.

Thus we must consider only the equations $(15,1), 2), 3)$ ). Skipping again most of the details, we get that

$$
a_{1}=\kappa b_{2}, \quad a_{2}=-\kappa b_{1}, \quad b_{1}=-\kappa a_{2}, \quad b_{2}=\kappa a_{1}
$$

where $\kappa= \pm 1$. We can choose the particular solution of these equations: $\kappa=1 ; a_{1}=N ; b_{1}=0$ and finally can get the expressions we wanted
1) $\boldsymbol{B}=r \cos \frac{u}{N} \boldsymbol{p}+N \sin \frac{u}{N}\left(\frac{\boldsymbol{x}}{r}-u \boldsymbol{p}\right)$
2) $\boldsymbol{C}=N\left(\frac{\boldsymbol{x}}{r}-u \boldsymbol{p}\right) \cos \frac{u}{N}-r \boldsymbol{p} \sin \frac{u}{N}$
3) $\boldsymbol{A}=N\left(\frac{1}{r}-p^{2}\right) \boldsymbol{x}+u N \boldsymbol{p}$
4) $\boldsymbol{L}=\boldsymbol{x} \times \boldsymbol{p}$
5) $Q=N\left(1-r p^{2}\right) \cos \frac{u}{N}-u \sin \frac{u}{N}$
6) $R=-u \cos \frac{u}{N}-N\left(1-r p^{2}\right) \sin \frac{u}{N}$
7) $S=N$. 
Let us recapitulate. We have found functions $\mu_{i j}$, such that their Poisson brackets can be considered as generators of the Lie algebra $\mathfrak{s o}(4,2)$. More precisely, $\mu_{i j}$ are the functions

$$
\begin{array}{ll}
\mu_{i 5}=B_{i} & i=1,2,3 \\
\mu_{45}=-Q & \\
\mu_{i 6}=C_{i} & i=1,2,3 \\
\mu_{46}=-R & \\
\mu_{i j}=p_{i} x_{j}-p_{j} x_{i} & i, j=1,2,3 \\
\mu_{i 4}=A_{i} & i=1,2,3 \\
\mu_{56}=S=N . &
\end{array}
$$

If we choose the parameters in different way, for example if we put $a_{1}=N$, $b_{1}=0, \kappa=-1$ we shall get

$$
\begin{aligned}
\boldsymbol{B} & =r \cos \frac{u}{N} \boldsymbol{p}+N \sin \frac{u}{N}\left(\frac{\boldsymbol{x}}{r}-u \boldsymbol{p}\right) \\
\boldsymbol{C} & =-N \cos \frac{u}{N}\left(\frac{\boldsymbol{x}}{r}-u \boldsymbol{p}\right)+r \sin \frac{u}{N} \boldsymbol{p} \\
Q & =-N\left(1-r p^{2}\right) \cos \frac{u}{N}-u \sin \frac{u}{N} \\
R & =u \cos \frac{u}{N}-N\left(1-r p^{2}\right) \sin \frac{u}{N} .
\end{aligned}
$$

(We have written here only those expressions differing from the previous ones). If we make the choice $a_{1}=N, b_{1}=0, \kappa=-1$, then it can be checked that the corresponding $\mu_{\lambda \nu}$ satisfy the relations

$$
\begin{array}{ll}
\mu_{\lambda 5} \mu_{\nu 6}-\mu_{\nu 5} \mu_{\lambda 6}=\mu_{56} \mu_{\lambda \nu} & \lambda, \nu=1,2,3,4 \\
\mu_{\lambda 5} \mu_{\lambda 5}=\mu_{\lambda 6} \mu_{\lambda 6}=\mu_{56}^{2} & \\
\mu_{\lambda 5} \mu_{\lambda 6}=0, & \mu_{56}<0
\end{array}
$$

(summation over repeated indeces assumed). It can be shown, that these relations determine the orbit, that is the orbit is defined by them as submanifold. We have put the proof of this fact in the next section.

The functions $\left(\mu_{\lambda 5}, \mu_{\lambda 6}\right) \equiv\left(\hat{Q}_{\lambda}, \hat{P}_{\lambda}\right)$ are the so-called Bacry-Györgyi variables and we can recover here some classical results about the Kepler's problem. For example, it can be checked that the map

$$
\psi:(\boldsymbol{x}, \boldsymbol{p}) \longrightarrow\left(\mu_{\lambda 5}, \mu_{\lambda 6}\right)=(\hat{Q}(\boldsymbol{x}, \boldsymbol{p}), \hat{P}(\boldsymbol{x}, \boldsymbol{p}))
$$


is an inclusion of $\mathcal{M}$ into a submanifold $\mathcal{N} \subset \mathbb{R}^{8}$, defined by

$$
\mathcal{N}=\{(\hat{Q}, \hat{P}) ;\langle\hat{Q}, \hat{Q}\rangle-\langle\hat{P}, \hat{P}\rangle=\langle\hat{Q}, \hat{P}\rangle=0, \hat{P} \neq 0\}
$$

where $\hat{Q}, \hat{P}$ are 4 -dimensional vectors, $\hat{Q} \equiv\left(\boldsymbol{Q}, Q_{4}\right)$ and $\langle\hat{Q}, \hat{P}\rangle$ stands for the standard scalar product in $\mathbb{R}^{4}$, defining the usual norm \|\| . From here one can obtain the famous result of Moser [18], as a matter of fact, the map

$$
\varphi:(\boldsymbol{x}, \boldsymbol{p}) \longrightarrow\left(\frac{\hat{P}(\boldsymbol{x}, \boldsymbol{p})}{\|\hat{P}(\boldsymbol{x}, \boldsymbol{p})\|}, \hat{Q}(\boldsymbol{x}, \boldsymbol{p})\right)
$$

is exactly the so called Lignon-Schaaf regularization map, see [5], which defines a symplectic immersion of $\mathcal{M}$ into $T^{+} \mathbb{S}^{3}$. It is clear that if $\psi$ regularizes the flow, $\varphi$ also has this property. This is easy to show for the map $\psi$. First of all however, recall that the flow of the Kepler's problem is not complete, since the trajectories with initial velocities directed to the center "fall" on it for finite time. This flow is avoided assuming that the phase space $\mathcal{M}$ is symplectically immersed in some other phase space, very lucidly it these ideas are discussed in the report [27] on [5] and also in [22]. In our case we have a natural immersion of $\mathcal{M}$ into $\mathcal{N}$ and the Hamiltonian flow is immersed into a complete flow. Indeed, for the Hamiltonian vector field $\xi_{H}$

$$
\left(\mathrm{d} \hat{Q}_{\lambda}\left(\xi_{H}\right), \mathrm{d} \hat{P}_{\lambda}\left(\xi_{H}\right)\right)=\left(\left\{Q_{\lambda}, H\right\},\left\{P_{\lambda}, H\right\}\right), \quad i_{\xi_{H}} \omega=-\mathrm{d} H
$$

we can write the evolution equations in terms of $(\hat{Q}, \hat{P})$ and check our statement. But it is easier to calculate the evolution, defined not by the $\xi_{H}$ but by the field $\eta$, corresponding to Hamiltonian function equal to $-S=-N$

$$
\left(\mathrm{d} \hat{Q}_{\lambda}(\eta), \mathrm{d} \hat{P}_{\lambda}(\eta)\right)=-\left(\left\{Q_{\lambda}, S\right\},\left\{P_{\lambda}, S\right\}\right), \quad i_{\eta} \omega=\mathrm{d} N .
$$

Since $N$ depends only on $H$, it is constant of motion and the integral curves for $H$ and $-N$ differ by a change of the parameter. We have

$$
\begin{aligned}
& \{S, \hat{Q}\}=(\{S, \boldsymbol{B}\},-\{S, Q\})=(\boldsymbol{C},-R)=\hat{P} \\
& \{S, \hat{P}\}=(\{S, \boldsymbol{C}\},-\{S, R\})=(-\boldsymbol{B}, Q)=-\hat{Q} .
\end{aligned}
$$

Thus the equations of motion are

$$
\frac{\mathrm{d}}{\mathrm{d} t} \hat{Q}=\hat{P}(t), \quad \frac{\mathrm{d}}{\mathrm{d} t} \hat{P}=-\hat{Q}(t) .
$$

The solutions exist for every $t$ and on $\mathcal{N}$ the flow is the flow of a four-dimensional oscillator. 


\section{The Orbit Corresponding to the Hydrogen Atom (Kepler's Problem)}

We shall show now how one can find the orbit in an explicit way, that is as a submanifold, defined by some equalities and inequalities.

We have already the components $\mu_{i j}$ of the momentum map, corresponding to the group $\mathrm{SO}_{e}(4,2)$. Let us now write it into the form

$$
\mu=\frac{1}{2} \sum_{i j} \mu_{i j} I_{i j}^{*}
$$

where $I_{i j}^{*}$ is the dual basis of the basis $I_{i j}$ for $\mathfrak{s o}(4,2)$ we have introduced in (9). Since $\mathfrak{s o}(4,2)$ is semisimple, there exists an invariant scalar product $\langle$, with the help of which one can identify the adjoint and coadjoint representation. We choose it to be $\langle X, Y\rangle=-\frac{1}{2} \operatorname{Tr}(X Y)$ and of course it is proportional to the Killing form of the algebra, [8]. Then

$$
\left\langle g X g^{-1}, Y\right\rangle=\langle\operatorname{Ad}(g) X, Y\rangle=\left\langle X, \operatorname{Ad}\left(g^{-1}\right) Y\right\rangle=\left\langle\operatorname{Ad}^{*}(g) X, Y\right\rangle .
$$

Using the equivalence between $\mathfrak{s o}(4,2)$ and $\mathfrak{s o}^{*}(4,2)$ we find for the dual basis $I_{i j}{ }^{*}=\gamma_{i} \gamma_{j} I_{i j}$. Here $\gamma_{i}$ are the same as in (9). Thus we can assume that

$$
\mu=\frac{1}{2} \sum_{i, j} \mu_{i j} \gamma_{i} \gamma_{j} I_{i j} .
$$

However, the equations (20) preserve their form if we put instead of $\mu_{i j}$ the expressions $\sigma_{i j}=\gamma_{i} \gamma_{j} \mu_{i j}$ - the coordinates of $\mu$ in the basis $I_{i j}$. Thus one must prove that these equations define unique orbit $\mathcal{O}_{\mu_{0}} \subset \mathfrak{s o}(4,2)\left(\mu_{0}\right.$ will be specified later) in the adjoint representation, that is

$$
\mu=\sum_{i<j} \sigma_{i j} I_{i j} \in \mathcal{O}_{\mu_{0}}
$$

exactly when $\sigma_{i j}$ satisfy (20). The element $\mu_{0}$ is obtained if we fix some values of $\boldsymbol{x}_{0}, \boldsymbol{p}_{0}$ :

$$
\mu_{0}=\sum_{i<j} \sigma_{i j}^{0} I_{i j}, \quad \sigma_{i j}^{0}=\gamma_{i} \gamma_{j} \mu_{i j}\left(\boldsymbol{x}_{\mathbf{0}}, \boldsymbol{p}_{\mathbf{0}}\right)
$$

We choose $\boldsymbol{x}_{0}=(1,0,0), \boldsymbol{p}_{0}=(0,1,0)$. Then

$$
\mu_{0}=-\left(e_{12}-e_{21}\right)+\left(e_{16}+e_{61}\right)-\left(e_{25}+e_{52}\right)+\left(e_{56}-e_{65}\right)
$$


and by definition

$$
\mathcal{O}_{\mu_{0}}=\left\{g \mu_{0} g^{-1} ; g \in \mathrm{SO}_{e}(4,2)\right\} .
$$

An arbitrary element from the orbit defines it uniquely, but to find a set of relations (equalities and inequalities) that determine the orbit orbit is by no means an easy task, as far as we know it is not known even if this is always possible. Here however we are quite lucky: it is easy to check that the matrix $\mu_{0}$ is idempotent, that is $\mu_{0}^{2}=0$. This property is obviously invariant under the coadjoint action, in other words all the elements $X$ from the orbit satisfy $X^{2}=0$. Let us put

$$
X=\left(\begin{array}{ll}
L & M \\
M^{t} & N
\end{array}\right)
$$

where

- $L$ is $4 \times 4$ matrix with components $l_{\mu \nu}: l_{\mu \nu}=-l_{\nu \mu}, \mu, \nu=1,2,3,4$

- $M$ is $4 \times 2$ matrix with components $M_{\nu 1}=a_{\nu}, M_{\nu 2}=b_{\nu}$

- $N$ is $2 \times 2$ matrix with components $N_{11}=N_{22}=0, N_{12}=-N_{21}=c$

We shall write also $X=\left(l_{\mu \nu}, a_{\nu}, b_{\nu}, c\right)$. The condition $X^{2}=0$ is equivalent to the equations

$$
\begin{aligned}
& \text { 1) } a_{\nu} a_{\nu}=b_{\nu} b_{\nu}=c^{2} \\
& \text { 2) } l_{\nu \rho} a_{\rho}=c b_{\rho} \\
& \text { 3) } l_{\nu \rho} b_{\rho}=-c a_{\rho} \\
& \text { 4) } a_{\nu} a_{\mu}+b_{\nu} b_{\mu}+l_{\nu \rho} l_{\rho \mu}=0
\end{aligned}
$$

(summation over repeated indices is assumed).

Note also that $c \neq 0$. Indeed, if the opposite is true then $X=0$. It is not hard to prove

Proposition 3. The system (36) is equivalent to the system

1) $a_{\nu} a_{\nu}=b_{\nu} b_{\nu}=c^{2}$

2) $l_{\nu \rho} a_{\rho}=c b_{\rho}$

3) $\quad l_{\nu \rho} b_{\rho}=-c a_{\rho}$

4) $c l_{\rho \nu}+a_{\rho} b_{\mu}-b_{\rho} a_{\mu}=0$.

Proof. Consider the linear operator $L: \mathbb{C}^{4} \mapsto \mathbb{C}^{4}$ and defined by the matrix $\left(l_{\mu \nu}\right)_{1 \leq \mu, \nu \leq 4}$. It is anti-Hermitian and hence semisimple. If $a=\left(a_{\mu}\right)_{1 \leq \mu \leq 4}, b=$ $\left(b_{\mu}\right)_{1 \leq \mu \leq 4}$ are treated as column vectors, the system (36) implies that $e_{1}=b+\mathrm{i} a$ 
and $e_{2}=b-\mathrm{i} a$ are the unique eigenvectors with eigenvalues $\pm \mathrm{i} c$ and that there are no other nonzero eigenvalues. From this the fourth equation in (37) follows immediately.

Proposition 4. The equations (37) define exactly two orbits of $\mathrm{SO}_{e}(4,2)$, one for $c>0$ and the other for $c<0$. The orbit $\mathcal{O}_{\mu_{0}}$ corresponds to $c<0$.

The proof is quite elementary, one uses the one-parametric subgroups of $\mathrm{SO}_{e}(4,2)$ in order to show that if $X_{1}, X_{2}$ are two points satisfying (37) with $c>0$ there exists an element $g \in \mathrm{SO}_{e}(4,2)$ such that $X_{2}=g X_{1} g^{-1}$.

Let us make one final remark. If we change the signature, that is if instead of ++++-- we choose ----++ we need just to change $I_{i j}$ into $-I_{i j}$. Then $\mu_{i j}$ must be changed to $-\mu_{i j}$ and finally one can remark that the orbit is the same but now $\mu_{56}>0$.

\section{The Orbit Corresponding to the Conformal Particle with Mass Zero, Spirality Zero}

In this section we shall choose the opposite signature for $\mathfrak{s o}(4,2)$, that is - - - ++ . The point is that we want the usual signature for the Poincaré group. Let us put

$$
\begin{gathered}
Z_{\mu}=I_{13+\mu}, \quad W_{\mu}=I_{23+\mu}, \quad L_{\mu \nu}=I_{3+\mu 3+\nu}, \quad S=I_{12} \\
\mu, \nu=0,1,2,3 .
\end{gathered}
$$

Then the commutation relations can be cast in the following form:

$$
\begin{array}{ll}
{\left[S, Z_{\mu}\right]=-W_{\mu},} & {\left[Z_{\mu}, Z_{\nu}\right]=-L_{\mu \nu}} \\
{\left[W_{\mu}, W_{\nu}\right]=-L_{\mu \nu},} & {\left[S, W_{\mu}\right]=Z_{\mu}} \\
{\left[Z_{\mu}, W_{\nu}\right]=\delta_{\mu \nu} S,} & {\left[W_{\mu}, L_{\lambda \nu}\right]=\delta_{\mu \nu} W_{\lambda}-\delta_{\mu \lambda} W_{\nu}} \\
{\left[S, L_{\mu \nu}\right]=0,} & {\left[Z_{\mu}, L_{\lambda \nu}\right]=\delta_{\nu \mu} Z_{\lambda}-\delta_{\mu \lambda} Z_{\nu}} \\
{\left[L_{\mu \nu}, L_{\kappa \lambda}\right]=\delta_{\mu \kappa} L_{\nu \lambda}+\delta_{\nu \lambda} L_{\mu \kappa}} & -\delta_{\mu \lambda} L_{\nu \kappa}-\delta_{\nu \kappa} L_{\mu \lambda} .
\end{array}
$$

A basis in the same algebra form also the vectors

$$
\begin{array}{cc}
X_{\mu \nu}=I_{2+\mu, 2+\nu}, \quad & T_{\mu}=I_{1,2+\mu}-I_{2+\mu, 6} \\
D=I_{16}, \quad & K_{\mu}=I_{1,2+\mu}+I_{2+\mu, 6} \\
\nu, \mu=1,2,3,4 .
\end{array}
$$


One can check that the set of generators $\left.\left\{X_{\mu \nu}, T_{\mu}\right)\right\}_{1 \leq \mu, \nu \leq 4}$ span the algebra of the Poincaré group and the same holds true for the generators $\left.\left\{X_{\mu \nu}, K_{\mu}\right)\right\}_{1 \leq \mu, \nu \leq 4}$. The commutation relations between the generators (40) run as follows

$$
\begin{gathered}
{\left[D, T_{\mu}\right]=-T_{\mu},\left[D, K_{\mu}\right]=K_{\mu}, \quad\left[D, X_{\mu \nu}\right]=0} \\
{\left[K_{\mu}, K_{\nu}\right]=0, \quad\left[T_{\mu}, T_{\nu}\right]=0, \quad\left[K_{\mu}, T_{\nu}\right]=-2\left(X_{\mu \nu}+\gamma_{\mu \nu} D\right)} \\
{\left[T_{\lambda}, X_{\mu \nu}\right]=\gamma_{\lambda \mu} T_{\nu}-\gamma_{\lambda \nu} T_{\mu},\left[K_{\lambda}, X_{\mu \nu}\right]=\gamma_{\lambda \mu} K_{\nu}-\gamma_{\lambda \nu} K_{\mu}} \\
{\left[X_{\kappa \lambda}, X_{\mu \nu}\right]=\gamma_{\kappa \nu} X_{\lambda \mu}+\gamma_{\lambda \mu} X_{\kappa \nu}-\gamma_{\kappa \mu} X_{\lambda \nu}-\gamma_{\lambda \nu} X_{\kappa \mu} .}
\end{gathered}
$$

Our task is to describe the phase space of the conformal particle with mass zero and spirality zero with respect to the connected component of the Poincare group, considered as a subgroup of the group $\mathrm{SO}_{e}(4,2)$. According to our concepts the phase space of a particle with a given dynamical group can be considered as an orbit in the coadjoint representation of this group. Then the values of such quantities as mass, spirality and so on must be equal to the values of some invariants of the coadjoint action, these specific invariants are discussed later. So the invariants are of primary importance for our approach and we must say something about how given a Lie algebra $\mathcal{G}$ one can find orbits, for which there will be some polynomial invariants (a polynomial functions in $\mu \in \mathcal{G}^{*}$ that are constant on the orbit). A class of polynomial invariants can be constructed using the universal enveloping algebra $U$ of $\mathcal{G}$. Recall that $U$ is defined as factor $T / I$ of the tensor algebra $T$ of $\mathcal{G}$ over the two-side ideal $I$, generated by the elements of the type $\xi \otimes \eta-\eta \otimes \xi-[\xi, \eta] ; \xi, \eta \in \mathcal{G}$. The multiplication sign in $U$ is usually omitted and if $\xi_{1}, \xi_{2}, \ldots, \xi_{n}$ is some basis of $\mathcal{G}$ then the Poincaré-Birkhoff-Witt theorem states that $U$ is generated by the products $\xi_{1}^{m_{1}} \xi_{2}^{m_{2}} \ldots \xi_{n}^{m_{n}}$, where $m_{i}$ are nonnegative integers. There is canonical isomorphism between $U$ (as vector spaces, but not as algebras) and the algebra $H[x]$ of the homogeneous polynomials on the variables $\left(x_{1}, x_{2}, \ldots, x_{n}\right)$. Let $\xi_{j}, 1 \leq j \leq n$ be some fixed basis in $\mathcal{G}$ and $\xi^{j}, 1 \leq j \leq n$ be the dual basis. Let $x_{j}, 1 \leq j \leq n$, be the coordinates of the vectors from $\mathcal{G}^{*}$ in the dual basis. The elements $S\left(\eta_{1}, \eta_{2}, \ldots, \eta_{k}\right)$, where by the symbol $S$ we denote the symmetrization with respect to all the indices of the product $\eta_{1} \eta_{2} \ldots \eta_{k}$ and each $\eta_{j}$ is one of the elements of the basis of $\mathcal{G}$, also form a basis in $U$. Then the isomorphism we are speaking about takes an the element $X \in U$ of the form

$$
X=\sum_{i_{1}, i_{2}, \ldots, i_{k}} a^{i_{1} i_{2} \ldots i_{k}} S\left(\xi_{i_{1}}, \xi_{i_{2}}, \ldots, \xi_{i_{k}}\right)
$$

into the polynomial

$$
P_{X}=\sum_{i_{1}, i_{2}, \ldots, i_{k}} a^{i_{1} i_{2} \ldots i_{k}} x_{i_{1}} x_{i_{2}} \ldots x_{i_{k}} .
$$


The above correspondence does not depend on the choice of the basis of the algebra so the isomorphism between $U$ and $H[x]$ is canonical. The isomorphism, as remarked already, is only between vector spaces, not between the algebras, but nevertheless it can be used when we are looking for equations, satisfied by the elements of the orbits as it allows to define an action $T_{g}$ of $G$ on $H[x]$, [25]. If $\mu=x_{i} \xi^{i} \in \mathcal{G}^{*}$ (here and below summation over repeated index is assumed), then $\operatorname{Ad}^{*}(g) \mu=y_{i}(g, x) \xi^{i}$, where $y_{i}=y_{i}(g, x)$ are some functions of $x_{i}$ and $g$. More specifically

$$
y_{i}(x, g)=\left\langle\xi_{i}, \operatorname{Ad}^{*}(g) \mu\right\rangle=\left\langle\operatorname{Ad}(g) \xi_{i}, \mu\right\rangle=[\operatorname{Ad}(g)]_{i}^{j} x_{j}
$$

where $\operatorname{Ad}(g) \xi_{i}=[\operatorname{Ad}(g)]_{i}^{j} \xi_{j}$. Then we set

$$
P_{X} \longrightarrow T_{g}\left(P_{X}\right)=\sum_{i_{1}, i_{2}, \ldots, i_{k}} a^{i_{1} i_{2} \ldots i_{k}} y_{i_{1}}(x, g) y_{i_{2}}(x, g) \ldots y_{i_{k}}(x, g) \text {. }
$$

If $\xi \in \mathcal{G}$ then $\left.\xi \mapsto \frac{\mathrm{d}}{\mathrm{d} t} T_{\exp t \xi}\left(P_{X}\right)\right|_{t=0} \equiv A_{\xi}\left(P_{X}\right)$ is an operator acting in the space of polynomials, defining a representation $\xi \mapsto A_{\xi}$ of $\mathcal{G}$. If the polynomial $P_{X}$ is invariant, then $A_{\xi}\left(P_{X}\right)=0$ for $\xi \in \mathcal{G}$. The action of $G$ and the representation of $\mathcal{G}$ can be defined also in $U$ (we shall denote them by the same letters) due to the canonical isomorphism between the two spaces. If $X$ is as in (42) then

$$
A_{\xi}(X)=\sum_{i_{1}, i_{2}, \ldots, i_{k}} a^{i_{1} i_{2} \ldots i_{k}}\left[\xi, S\left(\xi_{i_{1}}, \xi_{i_{2}}, \ldots, \xi_{i_{k}}\right)\right]
$$

where the bracket denotes the commutator. If the group is connected, the polynomial $P_{X}$ is invariant with respect to the above action of the group if and only if

$$
[\xi, X]=\xi X-X \xi=0, \quad \xi \in \mathcal{G}, \quad X \in U
$$

In other words, $X$ must be a Casimir operator. In what follows we identify the elements from $U$ and from $H[x]$, that is $X \in U$ and $P_{X} \in H[x]$ and denote them by the same letter - in each case it is easy to see what we have on mind.

As well-known, for the algebra of the Poincaré group there are two Casimir operators

$$
T^{2}=T_{\mu} T^{\mu}, \quad \omega^{2}=\omega_{\mu} \omega^{\mu}
$$

where $\omega_{\lambda}=\epsilon_{\lambda \rho \mu \nu} T_{\rho} X_{\mu \nu}$. (Here the usual rule for rising the indices is assumed, as well as a summation over repeated index). Then the orbits, for which $T^{2}=$ $m^{2} \neq 0, \omega^{2}=s(s+1)$ can be interpreted as the phase spaces of particles with mass $m$ and spin $s$. When $T^{2}=0$ the additional invariant is the spirality $\lambda$ which 
is introduced by the relation $\omega_{\mu}=\lambda T_{\mu}$. Therefore, if we are looking for the orbit corresponding to a particle with zero mass zero and spirality zero, its points must satisfy the equations

$$
T^{2}=0, \quad \omega^{2}=0 .
$$

However, when we have the Poincaré group realized as subgroup of the conformal group $\mathrm{SO}(4,2)$, the expressions $T^{2}$ and $\omega^{2}$ are no more Casimir operators for the algebra $\mathfrak{s o}(4,2)$ and the equations (47) generally speaking are not invariant. In more general setting, suppose we have some Lie group $G$ with algebra $\mathcal{G}$ and we have elements $l_{i} \in U$, (not necessarily Casimir operators), but we want the equations

$$
l_{i}=0, \quad l_{i} \in U, \quad i=1,2 \ldots s
$$

to be invariant, and the set of points that satisfy them to be union of orbits. It will certainly be so if $l_{i} \in U$ satisfy the requirements

$$
\left[\xi_{k}, l_{i}\right]=\sum_{j} a_{k i}^{j} l_{j}, \quad a_{k i}^{j} \in U .
$$

(In our case $T^{2}$ and $\omega^{2}$ must be among the elements $l_{i}$.) Our idea is now to find elements with the above property. Suppose we start with some elements $l_{j}, 1 \leq j \leq s_{0}$, and require that the equations $l_{i}=0$ (we remind here that we mean of course the corresponding polynomial equations) to be invariant (in our case these elements will be $T^{2}$ and $\omega^{2}$ ). Then we calculate commutators with the generators and obviously have two possibilities: i) the commutators are expressed through $\left\{l_{j}\right\}_{1 \leq j \leq s_{0}}$. Then we already have the situation we are looking for and we stop. ii) the commutators cannot be expressed through the elements $l_{j}$ but are expressed through $l_{j}$ and another linearly independent elements $m_{h}$, $1 \leq h \leq s_{1}$. Now, since the equations $l_{i}=0$ are invariant, then the equations $m_{h}=0$ will be invariant too. We must add those $m_{h}$ to our system and check again if the new system $\left\{l_{i}, m_{h}\right\}_{1 \leq i \leq s_{0} ; 1 \leq h \leq s_{1}}$ is closed under the commutators with the generators. The process must continue until we obtain a closed system (of course, if it is possible). This program does not seem very encouraging as it means a good deal of calculations. However, in our case it can be performed, so let us start with it.

Using the standard generators we have introduced, the operator $T^{2}$ can be expressed as follows

$$
T^{2}=S^{2}+W_{3}^{2}+Z_{3}^{2}+L_{3 \mu} L_{3 \mu}-Z_{\mu} Z_{\mu}-\left\{S, W_{3}\right\}
$$

where the summation over the repeated indices is assumed and the brackets $\{$, denote the anti-commutator. 
We start by the commutators of $T^{2}$ with the generators and we have

$$
\begin{array}{ll}
{\left[T^{2}, S\right]=F,} & {\left[T^{2}, Z_{\nu}\right]=F_{\nu}+\delta_{3 \nu} G} \\
{\left[T^{2}, W_{\nu}\right]=\delta_{3 \nu} F,} & {\left[T^{2}, L_{\nu \lambda}\right]=\delta_{3 \lambda} F_{\nu}-\delta_{3 \nu} F_{\lambda}}
\end{array}
$$

where

$$
\begin{aligned}
F & =\left\{W_{\mu}, L_{\mu 3}\right\}+\left\{S, Z_{3}\right\}-\left\{Z_{\mu}, W_{\mu}\right\} \\
F_{\nu} & =\left\{Z_{\mu}, L_{\mu \nu}\right\}-\left\{S, W_{\nu}\right\}+\left\{L_{\mu \nu}, L_{\mu 3}\right\}+\left\{Z_{\nu}, Z_{3}\right\}+\left\{W_{\nu}, W_{3}\right\} \\
G & =\left\{L_{\mu 3}, Z_{\mu}\right\}-\left\{W_{3}, S\right\}+\{S, S\}-\left\{Z_{\mu}, Z_{\mu}\right\} .
\end{aligned}
$$

So the elements $F, F_{\nu}(\nu \neq 3)$ and $G+F_{3}$ must be included in our system too. Next, calculating $\left[F_{3}+G, S\right],\left[F_{3}+G, Z_{\nu}\right],\left[F_{3}+G, L_{\kappa \lambda}\right],\left[F_{\mu}, S\right],\left[F_{\mu}, Z_{\nu}\right]$ $(\mu \neq 3),\left[F_{\mu}, W_{\nu}\right]$ and $\left[F_{\mu}, L_{\kappa \lambda}\right](\mu \neq 3)$, we add the elements

$$
\left\{L_{\mu \nu}, W_{\mu}\right\}+\left\{S, Z_{\nu}\right\}, \quad \nu=0,1,2,3 .
$$

Commuting these elements with $S$ yields additional elements

$$
\left\{L_{\mu \nu}, Z_{\mu}\right\}-\left\{S, W_{\nu}\right\}, \quad \nu=0,1,2,3
$$

and if we consider $[F, S]$, we see that in the system must be included also

$$
\left\{W_{\mu}, W_{\mu}\right\}-\left\{Z_{\mu}, Z_{\mu}\right\}
$$

Denote by $\Lambda_{\kappa \mu}$ the following elements

$$
\Lambda_{\kappa \mu}=\left\{L_{\kappa \rho}, L_{\rho \mu}\right\}+\left\{W_{\kappa}, W_{\mu}\right\}+\left\{Z_{\kappa}, Z_{\mu}\right\} .
$$

Then calculating $\left[F_{3}+G, L_{\kappa \lambda}\right]$ for $\kappa \neq 3, \lambda=3$ we get additional element $\Lambda_{\kappa 3}$ and considering $\left[F_{\mu}, Z_{\nu}\right]$ and $\left[F, W_{3}\right]$ we obtain the following set

$$
\begin{array}{ll}
\text { 1) } & \left\{L_{\mu \nu}, W_{\nu}\right\}+\left\{S, Z_{\nu}\right\} \\
\text { 2) } & \left\{L_{\mu \nu}, Z_{\nu}\right\}-\left\{S, W_{\nu}\right\} \\
\text { 3) } & \Lambda_{\mu \nu} \\
\text { 4) } & \Lambda_{\mu \mu}-\Lambda_{33} \\
\text { 5) } & \Lambda_{33}-\{S, S\}-\left\{Z_{\mu}, Z_{\mu}\right\} \\
\text { 6) } & \left\{Z_{\mu}, W_{\nu}\right\} \\
\text { 7) } & \left\{Z_{\mu}, Z_{\mu}\right\}-\left\{W_{\mu}, W_{\mu}\right\} .
\end{array}
$$

Commuting the above elements with the generators does not give new elements. 
Let us perform the same procedure calculating the commutators with $\omega_{\rho}$. We remind that

$$
\begin{array}{rlrl}
\omega_{0} & =\frac{1}{4} \epsilon_{i j k}\left\{T_{i}, X_{j k}\right\}, & i, j, k=1,2,3 \\
\omega_{i}=\frac{1}{4} \epsilon_{i j k}\left(2\left\{T_{j}, X_{0 k}\right\}-\left\{T_{0}, X_{j k}\right\}\right), & i, j, k=1,2,3
\end{array}
$$

where

$$
\begin{array}{ll}
T_{j}=Z_{j-1}-L_{j-1,3}, & T_{0}=S-W_{3} \\
X_{0 k}=W_{k-1}, & X_{j k}=L_{j-1, k-1} .
\end{array}
$$

Skipping the technical details, we obtain after commutation with the generators that the new elements which must be added to the system (53) are

$$
\left\{S, L_{\mu \nu}\right\}-\left\{Z_{\mu}, W_{\nu}\right\}+\left\{Z_{\nu}, W_{\mu}\right\}
$$

where the indices run over $0,1,2,3$. Thus the set we are looking for consists of the elements (53) and (54).

Let us consider now the corresponding polynomials. The scalar product $\langle X, Y\rangle=$ $-\frac{1}{2} \operatorname{Tr}(X Y)$ allows to identify $\mathfrak{s o}(4,2)$ and $\mathfrak{s o}(4,2)^{*}$, see Section 3 . If $I_{i j}$ is the basis for $\mathfrak{s o}(4,2)$ (with the signature adopted in this Section), then let $I_{i j}^{*}$ be the dual basis. According to the above identification it is a basis of $\mathfrak{s o}(4,2)$ too. If the components of a given element are $s, l_{\mu \nu}, w_{\mu \nu}$, let us denote by $s^{*}, l_{\mu \nu}^{*}, w_{\mu \nu}^{*}$ the components of the same element in the dual basis. One easily checks that

$$
s^{*}=s, \quad l_{\mu \nu}^{*}=l_{\mu \nu}, \quad z_{\mu}^{*}=-z_{\mu}, \quad w_{\mu}^{*}=-w_{\mu} .
$$

If $x \in \mathfrak{s o}(4,2)$ we will write

$$
x=s S+\frac{1}{2} l_{\mu \nu} L_{\mu \nu}+z_{\mu} Z_{\mu}+w_{\mu} W_{\mu}
$$

or in other words $s, l_{\mu \nu}=-l_{\mu \nu}, z_{\mu}, w_{\mu}, \mu, \nu=0,1,2,3$ are the components of the element $x \in \mathfrak{s o}(4,2)$ in the basis we have chosen. In the dual basis

$$
x=s S^{*}+\frac{1}{2} l_{\mu \nu} L_{\mu \nu}^{*}-z_{\mu} Z_{\mu}^{*}-w_{\mu} W_{\mu}^{*} .
$$

It follows that the polynomials, corresponding to the elements from the system of 
the elements (53) and (54) are

1) $s l_{\mu \nu}-z_{\mu} w_{\nu}+z_{\nu} w_{\mu}$

2) $s z_{\nu}-l_{\mu \nu} w_{\mu}$

3) $-s z_{\nu}-l_{\mu \nu} w_{\mu}$

4) $l_{\kappa \rho} l_{\rho \mu}+w_{\kappa} w_{\mu}+z_{\kappa} z_{\mu}$

5) $l_{\mu \rho} l_{\rho \mu}+w_{\mu} w_{\mu}+z_{\mu} z_{\mu}-l_{3 \rho} l_{\rho 3}-w_{3} w_{3}-z_{3} z_{3}$

6) $l_{3 \rho} l_{\rho 3}+w_{3} w_{3}+z_{3} z_{3}-s^{2}-z_{\mu} z_{\mu}$

7) $w_{\mu} z_{\mu}$

8) $z_{\mu} z_{\mu}-w_{\mu} w_{\mu}$

(there is no summation over $\mu$ in 5)).

Evaluated on the orbit, we are looking for, these polynomials must be equal to zero. It is not hard to see that we can select the following independent equations

$$
\begin{aligned}
& \text { 1) } s l_{\mu \nu}=z_{\mu} w_{\nu}-z_{\nu} w_{\mu} \\
& \text { 2) } z_{\mu} z_{\mu}-w_{\mu} w_{\mu}=0 \\
& \text { 3) } w_{\mu} z_{\mu}=0 \\
& \text { 4) } z_{\mu} z_{\mu}=s^{2} .
\end{aligned}
$$

These equations coincide with the equations we have obtained earlier considering the Kepler's problem. We know that they define two orbits - one for $s>0$ and another for $s<0$. In order to decide which of these orbits we must choose, we have to remark that $T_{0}=s-w_{3}$. As in addition $w_{\mu} w_{\mu}=s^{2}$, then $\left|w_{3}\right|<|s|$ and $T_{0}$ and $s$ have the same sign. According to the general recipe for obtaining the phase spaces for Poincaré-type particles one must have $T_{0}>0$ to ensure that the linear momentum $T$ is future-like vector. Therefore the orbit we are looking for corresponds to the choice $s>0$. Now taking into account the necessary change of signs when one passes to the opposite signature, we can formulate our main result:

Theorem 5. The Kepler's problem (Hydrogen atom problem) phase space and the phase space of a conformal particle with mass zero and spirality zero are identical at the classical level. 


\section{Acknowledgements}

Most of the material included in the present article is as a matter of fact included in my diploma work. Naturally, I am indebted to all colleagues that helped me at that time and to my former supervisor Ivan Todorov. However, none of the results from the diploma work have been ever published. I am greatly indebted to Ivailo Mladenov, who pointed out to me that some of them deserve better fate and prompted me to write the present work.

\section{References}

[1] Abraham R. and Marsden J., Foundations of Mechanics, Reading MA, Benjamin/Cummings, 2-nd Edition, 1978.

[2] Bacry H., The de Sitter Group $L_{41}$ and the Bound States of Hydrogen Atom, Nouvo Cimento A 41 (1966) 222-234.

[3] Barut A. and Bornzin G., Formulation of the Symmetry Breaking in Relativistic Kepler Problems with or without Magnetic Charges. J. Math. Phys. 12 (1971) 841-846.

[4] Cordani B., The Kepler Problem: Group Theoretical Aspects, Regularization and Quantization, with Applications to the Study of Perturbations, Birkhäuser, Basel, 2002.

[5] Cushman R. and Bates L., Global Aspects of Classical Integrable Systems, Birkhäuser, Basel, 1997.

[6] Györgyi G., Integration of the Dynamical Symmetry Group for the $-\frac{1}{r}$ Potential, Acta Phys. Acad. Sci. Hungar. 27 (1969) 435-439.

[7] Györgyi G., Kepler's Equation, Fock's Variables, Bacry's Generators and Dirac Brackets, Nuovo Cimento A 62 (1969) 449-473.

[8] Goto M. and Grosshans F., Semisimple Lie Algebras, Lecture Notes in Pure and Applied Mathematics 38, M. Dekker, New York, 1978.

[9] Guillemin V. and Sternberg S., Geometric Asymptotics, American Mathematical Society, Providence, RI, 1977.

[10] Iosifescu M. and Scutaru H., On Six-Dimensional Canonical Realisations of the $\mathfrak{s o}(4,2)$ Algebra, J. Math Phys. 21 (1980) 2033-2045.

[11] Iwai T., A Dynamical Group $S U(2,2)$ and its Use in the MIC-Kepler Problem, J. Phys. A: Math. Gen. 26 (1993) 609-630. 
[12] Kirillov A., Elements of Representation Theory (in Russian), Nauka, Moscow, 1970.

[13] Kostant B., Quantization and Unitary Representations, Lecture Notes in Mathematics, 170, C. T. Taam, Ed., Springer, New York, 1970.

[14] Kummer M., On the Regularizations of Kepler's Problem, Comm. Math. Phys. 84 (1982) 133-152.

[15] Mack G. and Todorov I., Irreduicibility of the Ladder Representation of $U(2,2)$ when Restricted to the Poincaré Subgroup, J. Math. Phys. 10 (1969) 2078-2085.

[16] McAnally D. and Bracken A., Time Dependence of Variables in the Quantum-Mechanical and Classical Coulomb Problems, and the Dynamical Algebra so (4,2), Phys. Rev. A 37 (1988) 2304-2308.

[17] Mladenov I. and Tsanov, V. Geometric Quantisation of the MIC-Kepler Problem, J. Phys. A 20 (1987) 5865-5871.

[18] Moser J., Regularization of Kepler's Problem and the Averaging Method on Manifold, Comm. Pure Appl. Math. 23 (1970) 609-636.

[19] Onofri E., Dynamical Quantization of the Kepler Manifold, J. Math. Phys. 17 (1976) 401-408.

[20] Souriau J.-M., Structure des Systèmes Dynamiques, Dunod, Paris, 1970.

[21] Souriau J.-M., Sur la Varieté de Kepler, Simposia Mathematica XIV, Academic Press, London, 1974.

[22] Souriau J.-M., On the Kepler Manifold, Preprint, translation from French. math.rice.edu/ jorgen/TeX_Docs/Plain/Kepler.ps

[23] Todorov I., Sur la quantification d'une système Mécanique avec des constraintes de deuxième classe, Preprint. IHES, 1976.

[24] Tripathy K., Gupta R. and Anand J., O(4,2) Symmetry and the Classical Kepler Problem, J. Math. Phys. 16 (1968) 1139-1134.

[25] Trofimov V. and Fomenko A., Algebra and Geometry of the Integrable Hamiltonian Differential Equations, Factorial, Minsk, 1995.

[26] Tsanov V. and Mladenov I., Geometric Quantization of the Kepler Problem with a Magnetic Charge, In: Group Theoretical Methods in Physics, Proc. XVI-th Int. Colloq., Varna (Bulgaria) 1987, Lect. Notes Phys. 313 (1988) 275-281. 
[27] Tang X., Kepler Problem and SO(4) Momentum Map, math.berkeley.edu/alanw/277papers00/tang.pdf

Alexander B. Yanovski

Dept. Math. \& Appl. Mathematics

University of Cape Town

Rondebosch 7700

Cape Town

South Africa

E-mail: yanovski@maths.uct.ac.za 